\title{
PROCESOS HIDROLÓGICOS EN UNA CUENCA FORESTAL DEL SISTEMA CENTRAL: CUENCA EXPERIMENTAL DE RINCONADA
}

\author{
J. MARTÍNEZ FERNÁNDEZ, A. CEBALLOS BARBANCHO, V. HERNÁNDEZ \\ SANTANA, S. CASADO LEDESMA y C. MORÁN TEJEDA
}

\author{
Departamento de Geografía. Universidad de Salamanca
} C/ Cervantes, 3. 37002-Salamanca

Correo electrónico de contacto: jmf@usal.es

\begin{abstract}
RESUMEN. En este trabajo se analizan los procesos hidrológicos que caracterizan a una cuenca forestal de la vertiente norte del Sistema Central. La cuenca experimental de Rinconada (Rinconada de la Sierra, prov. de Salamanca), en funcionamiento desde el otoño de 1999, está ocupada en su mayor parte por un bosque denso de roble melojo. Cuenta con diferentes dispositivos (estaciones meteorológica e hidrológica automáticas, red de estaciones de medición de la humedad del suelo, parcelas de control de la dinámica hídrica vegetal, redes de control de la interceptación) para el seguimiento de variables que intervienen en el sistema suelo-agua-planta-atmósfera. Los resultados del análisis han puesto de manifiesto el papel tan destacado que tiene el bosque en la dinámica hidrológica. La cuenca tiene una baja capacidad de generar escorrentía, sobre todo en el periodo coincidente con la actividad biológica del roble melojo. Factores como la escasez de precipitaciones estivales, la elevada evapotranspiración, la interceptación y el consumo de agua de los árboles, determinan que la cuenca produzca muy poca escorrentía, a pesar de que se encuentre bajo condiciones climáticas subhúmedas. Se destaca, también, que las superficies saturadas, aun ocupando un porcentaje muy reducido de la cuenca, son determinantes en la generación de escorrentía de una gran mayoría de eventos.
\end{abstract}

ABSTRACT. In the present work we analyse the hydrological processes characterising a forested catchment on the northern face of the Sistema Central range. The Rinconada experimental catchment (at Rinconada de la Sierra, province of Salamanca), which has been running since autumn 1999, is mainly occupied by a dense forest of semi-deciduous oak (Quercus pyrenaica Willd.). It is equipped with different experimental devices (automatic meteorological and hydrological stations, a soil moisture measurement network, 
plots to monitor plant water dynamics, interception control networks) for the monitoring of the variables involved in the soil-water-plant-atmosphere system. The results of the analysis reveal the crucial role of the forest in the hydrological dynamics of the catchment. The catchment has a low capacity to generate runoff, above all during the period coinciding with the biological activity of the oaks. Factors such as low summer rainfall, high evapotranspiration, interception and water consumption by the trees mean that the catchment produces very little runoff, even though it is subject to subhumid climatic conditions. Also important is the fact that the saturated areas, even though occupying a very reduced percentage of the catchment, are determinant in the generation of the runoff from the majority of the events.

Palabras clave: generación de escorrentía, humedad del suelo, Quercus pyrenaica, déficit hídrico, interceptación, área saturada, clima mediterráneo.

Key words: runoff yield, soil moisture, Quercus pyrenaica, water deficit, interception, saturated area, Mediterranean climate.

Enviado el 27 de mayo de 2005

Aceptado el 15 de julio de 2005

\section{Introducción}

El análisis de los procesos hidrológicos en cuencas forestales es objeto de un interés creciente por parte de la comunidad científica desde múltiples y muy diversas disciplinas. Los cambios ambientales que se están observando en muchas regiones del Planeta, así como las transformaciones que están experimentando los usos del suelo en ámbitos geográficos muy diversos, están suscitando un gran número de investigaciones que tienen por objeto analizar las consecuencias de dichos cambios en medios tan sensibles como los forestales. En ese contexto, uno de los aspectos de mayor interés es el del binomio agua-bosque.

El papel del bosque en los procesos hidrológicos ha sido abordado desde muy diversos puntos de vista. Es bien conocida la reducción de la escorrentía que se produce como resultado de la intervención de factores como la interceptación o el consumo de agua por los árboles (Bosch y Hewlett 1982; Andréassian, 2004). En zonas donde se ha producido una recuperación del bosque, la generación de escorrentía se ha visto seriamente afectada (Rambal, 1987; Beschta et al., 2000). En áreas montañosas donde los cambios en el uso del suelo han propiciado una recuperación del bosque, se ha observado una apreciable disminución de la escorrentía superficial (Beguería et al., 2003). Más específicamente, en ambientes mediterráneos se ha detectado que en los suelos bajo cobertura arbórea se genera menos escorrentía superficial (Joffre y Rambal, 1993). Al mismo tiempo, en regiones donde se observa un proceso de deforestación (Bent, 2001) y una expansión de las áreas urbanas (Sun et al., 2004), se detecta un incremento en la producción y exportación de agua. La reducción de la cubierta vegetal ha provocado, en 
determinados ambientes, cambios en la dinámica estacional de la escorrentía y un incremento de los caudales máximos (Iroumé et al., 2005). De cualquier modo, las interacciones que están presentes en la dinámica hidrológica de las zonas forestales son de tal complejidad, que en la mayoría de los casos es difícil extraer conclusiones simples (Cosandey et al., 2005).

Durante las últimas décadas se viene observando en España un incremento notable, y en algunos casos espectacular, de la superficie ocupada por el bosque. Esto se ha puesto de manifiesto en los resultados de los sucesivos inventarios forestales nacionales (MMA, 2000). Se trata de un fenómeno sin precedentes con importantes repercusiones ecológicas, botánicas, paisajísticas, hidrológicas y, en general, medioambientales. El estudio de los cambios en los procesos hidrológicos de las zonas afectadas por el aumento de la superficie forestal se está centrando en determinadas regiones, mientras que en otras la información disponible es muy escasa. La cuenca del Ebro y, más concretamente, la vertiente pirenaica, es una de esas áreas donde se está estudiando con más profundidad este aspecto. En esa zona se ha observado, por ejemplo, que el incremento de la superficie forestal en las cuencas de cabecera, aun siendo moderado, ha dado lugar a una disminución significativa en la generación de escorrentía (Gallart y Llorens, 2004). El abandono de tierras de cultivo y la posterior recuperación de la cubierta vegetal en las montañas del Pirineo, está provocando cambios notables en el comportamiento hidrológico de ese territorio (García-Ruiz et al., 2005).

En otras zonas, donde se vienen detectando cambios similares a los ya señalados, como es el caso del Sistema Central, los estudios llevados a cabo y la información disponible son muy escasos. Motivado por esta circunstancia, el Grupo de Investigación en Recursos Hídricos de la Universidad de Salamanca instaló y equipó la Cuenca Experimental de Rinconada (Sierra de Tamames, Prov. de Salamanca) en el verano de 1999. El objetivo de esta iniciativa era contar con un dispositivo experimental permanente en una cuenca representativa de un amplio sector montañoso, bajo condiciones ambientales mediterráneas sub-húmedas, en donde se pudiera llevar a cabo un seguimiento de los procesos que definen su comportamiento hidrológico. En este trabajo se exponen los resultados de cinco años de experimentación, centrados, básicamente, en aspectos de la interacción del bosque con los procesos hidrológicos y en el análisis de los mecanismos de generación de escorrentía de la cuenca.

\section{Zona de estudio y equipamiento experimental}

La Cuenca Experimental de Rinconada (Fig. 1) tiene una superficie de 62.3 ha., entre 1190 y $1454 \mathrm{~m}$ de altitud y una pendiente media del 26\%. Está localizada (Foto 1) en la vertiente N del Sistema Central (Sierra de Tamames, prov. Salamanca). Las condiciones climáticas de la zona, definidas en la estación Rinconada de la Sierra (Instituto Nacional de Meteorología), situada al pie de la cuenca a $1000 \mathrm{~m}$ de altitud, son de tipo mediterráneo subhúmedo con una temperatura media de $10^{\circ} \mathrm{C}$ y una precipitación media anual de $953 \mathrm{~mm}$. La evapotranspiración potencial anual es de $853 \mathrm{~mm}$. El máximo de 


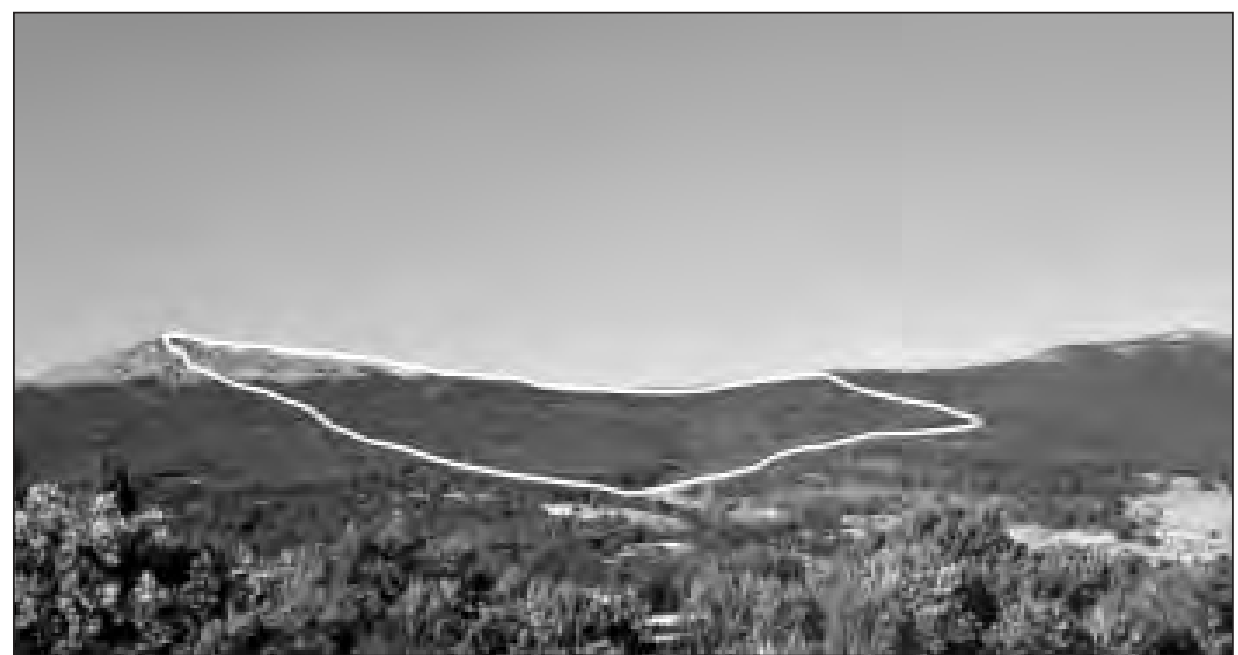

Foto 1. Ubicación de la Cuenca Experimental de Rinconada en la vertiente norte de la Sierra de Tamames (Prov. Salamanca).

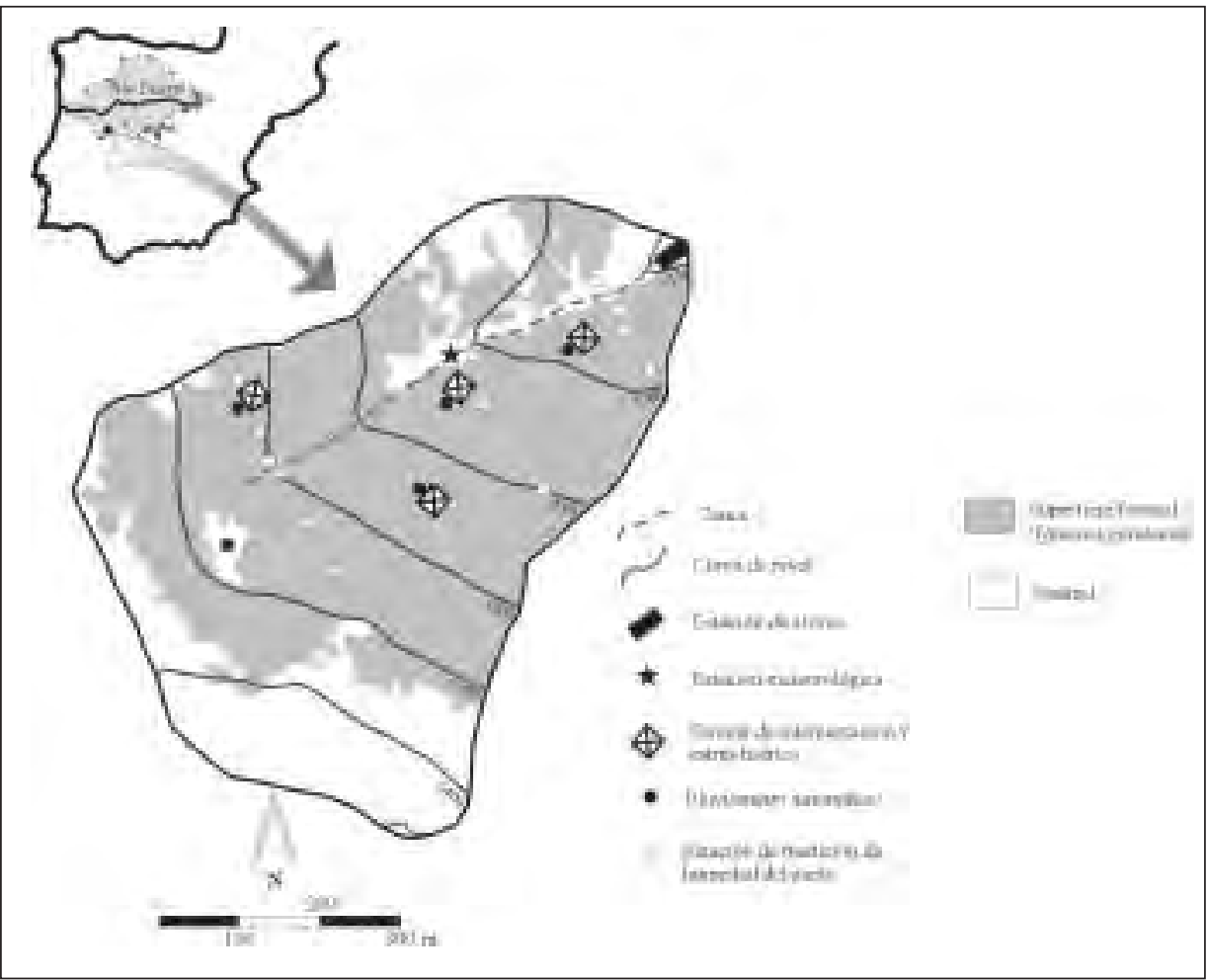

Figura 1. Localización y dispositivos experimentales de la cuenca experimental de Rinconada. 
precipitación mensual es de $130 \mathrm{~mm}$ en noviembre y el mínimo es de $15 \mathrm{~mm}$ en agosto. Se trata de una cuenca forestal, cubierta en un $68 \%$ por un bosque de roble melojo (Quercus pyrenaica Willd.) con una elevada densidad de individuos (2300 pies/ha).

El sustrato geológico está formado por una sucesión de bandas de cuarcitas, calizas, areniscas y pizarras, entre la divisoria y el piedemonte. La presencia de una formación de caliza paleozoica ha dado lugar a la formación de un acuífero y a la existencia de surgencias que manan en la propia cuenca. Los suelos de la C.E. de Rinconada poseen una textura fina, fundamentalmente limosa. Las fracciones limo y arcilla suponen entre el $70-80 \%$ de la matriz del suelo. El contenido de materia orgánica es elevado, en torno al $10 \%$ en los primeros $5-15 \mathrm{~cm}$, y por debajo del $1 \%$ más allá de los $25 \mathrm{~cm}$. Los suelos más comunes son leptosoles, cambisoles y regosoles, eútricos si están sobre pizarras y calizas, y dístricos si se desarrollan sobre cuarcitas y areniscas.

En la actualidad el único uso del suelo es el pastoreo extensivo. No obstante, en el pasado todos los montes de la zona estuvieron sometidos a una intensa explotación. La existencia de una mayor población y su dependencia de los recursos naturales del entorno más próximo, provocó que el bosque se explotara hasta el límite de hacerlo desaparecer periódicamente. Actividades como la ganadería, la explotación maderera, el carboneo, la fabricación de cal o la extracción de leña para el uso doméstico, mantuvieron sobre amplios sectores forestales una presión intensa y constante. Sin embargo, desde los años 70 del siglo pasado dicha presión se ha reducido considerablemente debido a factores demográficos, económicos y tecnológicos. En la C.E. de Rinconada, la última tala intensiva (a mata rasa) se produjo a principios de dicha década y el bosque actual es el resultado de la regeneración posterior y la escasa presencia de actividades humanas en el mismo.

En la cuenca experimental de Rinconada hay instalada desde octubre de 1999 una estación meteorológica automática para la medición de las principales variables climáticas (tempertura del aire, humedad relativa, déficit de presión de vapor, radiación global, velocidad y dirección del viento, y precipitación) que se registran cada 10 minutos. Desde esa misma fecha, la cuenca cuenta con una estación de aforos para el control de la escorrentía superficial en su extremo inferior. La estación hidrológica está compuesta por un vertedero tipo $\mathrm{H}$, en el que se mide la altura de la lámina de agua mediante un limnígrafo automático que registra el dato medio cada 10 minutos. Esta estación y la meteorológica están sincronizadas.

En la primavera de 2000 se instaló en la C.E. de Rinconada una red de 18 estaciones de medición de la humedad del suelo. Las red está compuesta por tres transectos de 6 estaciones cada uno, dispuestos perpendicularmente al cauce principal de la cuenca (Fig. 1). De esta forma en cada vertiente existe al menos una estación en cada posición de la ladera: divisoria, media ladera y pie de ladera. Cada estación está compuesta por un perfil de suelo equipado con sondas TDR (Time Domain Reflectometry) de dos varillas (Martínez Fernández y Ceballos, 2001) insertadas horizontalmente a 5, 15, 25, 50 y $100 \mathrm{~cm}$ de profundidad. Las sondas fueron introducidas en la pared inalterada del perfil excavado y se colocaron horizontalmente, paralelas a la dirección de la máxima pen- 
diente de la ladera. Una vez instaladas, el perfil fue restaurado, evitando al máximo la perturbación del suelo. La red se completó en abril de 2000 y desde entonces se han realizado mediciones de la humedad del suelo en cada estación cada dos semanas.

Para llevar a cabo las mediciones mediante el método TDR, se emplea un ecómetro Tektronix 1502C como generador de ondas electromagnéticas. Las ondas se analizan visualmente en el campo, siguiendo el método descrito por Cassel et al. (1994) y el contenido de agua del suelo se obtiene usando la fórmula propuesta por Topp et al. (1980). Previamente a las mediciones en el campo, el método fue validado en laboratorio (Martínez Fernández y Ceballos, 2001) mediante monolitos de suelo inalterado. Para verificar la aplicabilidad de la fórmula se utilizó como método de referencia el gravimétrico, tal y como sugieren Zegelin et al. (1992).

La interceptación se estima en cuatro parcelas de 20x20 m instaladas en marzo de 2004, representativas de diferentes estados del robledal de la cuenca, en cada una de las cuales hay instalados 16 pluviómetros totalizadores y uno automático. Con anterioridad (julio de 2002) se habían colocado cuatro dispositivos (uno por parcela) que consistían en un pluviómetro totalizador y un colector de escorrentía cortical. Como referencia para la medición de la precipitación fuera del dosel del bosque, se instalaron dos parejas de pluviómetros (uno totalizador y otro automático) en sendos claros situados en la parte alta y media de la cuenca, respectivamente. La dinámica suelo-agua-bosque se analiza desde junio de 2003 en estas mismas parcelas. El contenido de agua de los árboles y del suelo en la zona radicular se mide mediante TDR en cuatro individuos seleccionados por su representatividad dentro de cada parcela (Hernández Santana et al., 2004).

\section{Resultados y discusión}

\subsection{La mediterraneidad de la C.E. de Rinconada}

Desde un punto de vista estrictamente climático, los rasgos básicos del clima mediterráneo son reconocibles en el contexto geográfico de la C.E. de Rinconada, tras analizar la serie termopluviométrica correspondiente al observatorio del INM ubicado en el municipio de Rinconada de la Sierra $(1951-2004, \mathrm{n}=54)$.

A pesar de que la precipitación media anual de la cuenca sea de $953 \mathrm{~mm}$, el déficit pluviométrico durante el verano es apreciable, ya que los tres meses estivales (junio, julio y agosto) registran menos del $9 \%$ anual. Otro rasgo significativo de las precipitaciones en la zona de estudio es su apreciable variabilidad intra-anual. Si se admite 30 mm como un umbral válido para definir un mes como seco en el contexto de la Península Ibérica (López Gómez, 1989), en la zona se registra un promedio de 4.3 meses secos al año. Esta variabilidad intra-anual queda reflejada en el dato medio de la serie, correspondiente al Índice de Concentración de las Precipitaciones (De Luis et al., 2000), que con un valor de 16.9 permite afirmar que la C.E. de Rinconada acusa una estacionalidad media-alta en la distribución intra-anual de la lluvia. 
La serie pluviométrica también se caracteriza por su apreciable variabilidad interanual, con un coeficiente de variación del $26.7 \%$ y unos valores correspondientes al año más húmedo y al más seco de la serie, muy alejados de la media del período: 1528 y 489 $\mathrm{mm}$ respectivamente. Esta variabilidad inter-anual es perceptible en los 5 años de datos obtenidos en la C.E. de Rinconada (tabla 1). Aplicando el cálculo de quintiles propuesto por el Instituto Nacional de Meteorología (INM, 1994) para clasificar los distintos años en clases según el volumen de agua precipitada, los 5 totales anuales se distribuyen en 3 clases muy contrastadas: "muy seco", "muy húmedo" y "normal".

Los resultados del análisis de las rachas secas (Ceballos et al., 2004) confirman la mediterraneidad del área de estudio, ya que variables como el porcentaje de días secos al año $(>76 \%)$, la duración media del total de rachas ( $\approx 9$ días) y la duración media de las rachas máximas anuales $(\approx 44$ días), son comparables a las registradas en áreas netamente mediterráneas del sur y este de España (Martín-Vide y Gómez, 1999).

Finalmente, otro rasgo indicador de cierta mediterraneidad en la cuenca es la intensidad de la precipitación en 24 horas, 30 y 10 minutos (tabla 1). Sin alcanzar el carácter torrencial de la costa mediterránea, las intensidades medidas son superiores a las registradas en otras cuencas experimentales del ámbito mediterráneo (Schnabel y Mateos, 2000) ubicadas en la mitad occidental de la Península Ibérica.

Tabla 1. Características de la lluvia en la cuenca experimental de Rinconada (2000-2004).

\begin{tabular}{|c|c|c|c|c|l|}
\hline Año & $\begin{array}{c}\text { Precipitación } \\
(\mathrm{mm})\end{array}$ & $\begin{array}{c}\text { Int. máx. 24 h } \\
(\mathrm{mm})\end{array}$ & $\begin{array}{c}\text { Int. máx. 10 } \\
\min \left(\mathrm{mm} \mathrm{h}^{-1}\right)\end{array}$ & $\begin{array}{c}\text { Int. máx. 30 } \\
\min \left(\mathrm{mm} \mathrm{h}^{-1}\right)\end{array}$ & $\begin{array}{c}\text { Quintiles } \\
(\mathrm{INM})\end{array}$ \\
\hline 2000 & 1038,0 & 87,6 & 19,2 & 15,6 & Normal \\
2001 & 976,6 & 60,6 & 43,2 & 32,0 & Normal \\
2002 & 1291,4 & 71,0 & 44,4 & 27,2 & Muy húmedo \\
2003 & 1215,6 & 101,0 & 66,0 & 44,0 & Muy húmedo \\
2004 & 649,6 & 42,9 & 61,2 & 50,4 & Muy seco \\
\hline
\end{tabular}

\subsection{Bosque y procesos hidrológicos}

La mediterraneidad del sector montañoso en el que se enmarca la C.E. de Rinconada se ve reflejada en los procesos hidrológicos que están más relacionados con el balance de radiación. Al mismo tiempo, la dinámica vegetal viene condicionada por una clara estacionalidad fenológica. La actividad biológica, identificada por la presencia de biomasa foliar, se circunscribe al periodo que va desde la segunda quincena de mayo a mediados de octubre. Ambas circunstancias se reflejan en un marcado contraste entre el periodo húmedo y frío, en el que el bosque está en parada biológica, y el templadocálido y seco que coincide con el periodo de actividad vegetativa del roble melojo. 


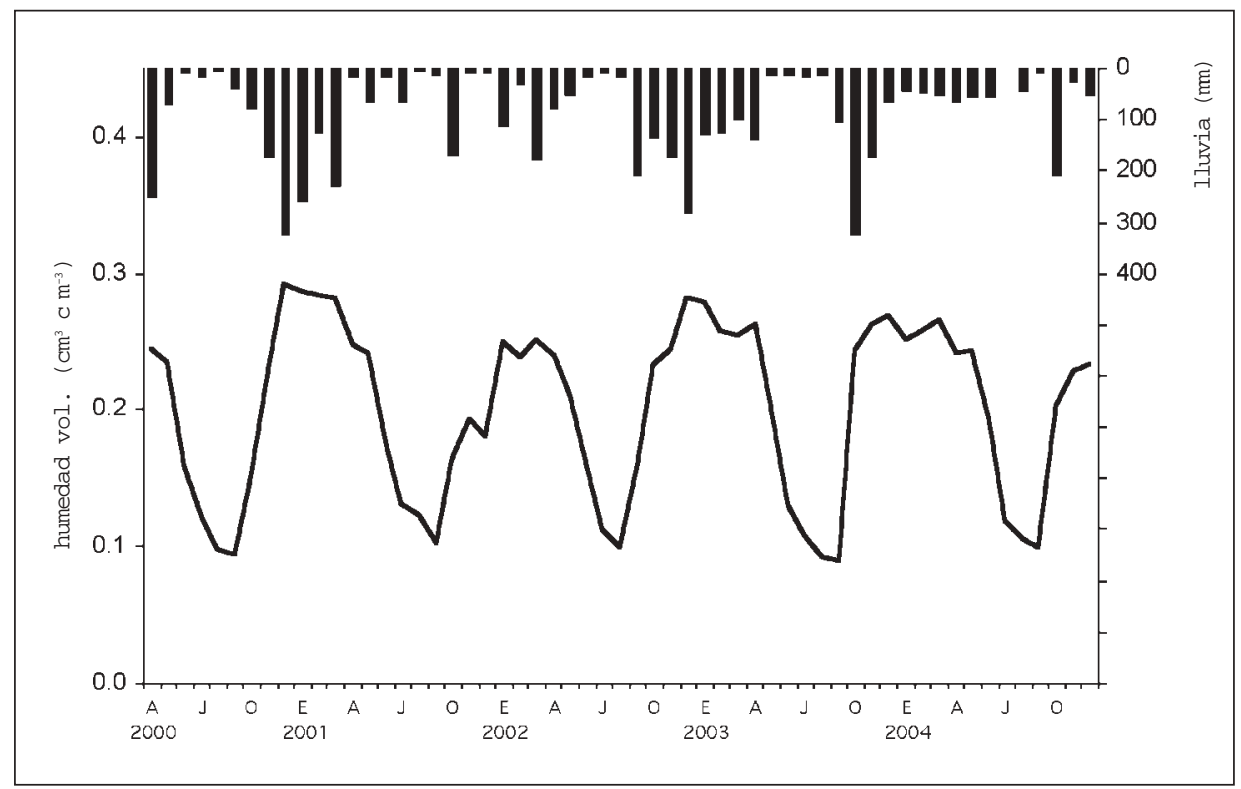

Figura 2. Evolución mensual de la precipitación y el contenido de agua del suelo en la C.E. de Rinconada durante el periodo abril-2000 y diciembre-2004.

Un buen indicador de esta dinámica dual es el contenido de agua del suelo, ya que es en si mismo un nexo entre vegetación y procesos hidrológicos. Los datos del registro de dicha variable muestran un enorme contraste estacional (Fig. 2). El hecho de que la precipitación en la zona de estudio sea abundante no es óbice para que durante los meses estivales el contenido de agua del suelo sea muy escaso. En los cinco años de funcionamiento de la cuenca experimental se ha medido en la estación meteorológica automática un promedio de 1036 mm de precipitación. Sin embargo, esa abundancia pluviométrica no impide que durante los meses estivales se alcance un acusado déficit hídrico.

Son varios los factores que están implicados en ese comportamiento. Durante el periodo que coincide con el ciclo vegetativo del bosque, se produce una relativa reducción en la cantidad lluvia (186 mm de promedio de mayo a septiembre entre 2000 y 2004). Un factor más decisivo es la elevada evapotranspiración que se registra durante ese periodo. De los $850 \mathrm{~mm}$ de ETP anual, $635 \mathrm{~mm}$ (el 75\%) se registran en esos cinco meses. Un tercer elemento a tener en consideración es la interceptación de la vegetación. Durante los años 2003-2004 se ha estimado que el porcentaje de precipitación interceptada por el robledal ha sido del $17 \%$ en la época invernal y del $32 \%$ en el verano. Las pérdidas por evapotranspiración y la merma de precipitación por la interceptación contribuyen decisivamente para que el suelo presente un acusado déficit hídrico.

Para entender mejor la significación de ese estado deficitario, resulta útil el análisis del Índice de Déficit Hídrico (IDH), que viene definido por la siguiente fórmula: 


$$
I D H=\left(\frac{\theta-\theta_{f c}}{\theta_{\mathrm{d}}}\right) \times 100
$$

donde $\theta$ es el contenido de humedad del suelo en un momento dado, $\theta_{\mathrm{d}}$ es la diferencia entre la humedad a capacidad de campo $\left(\theta_{\mathrm{fc}}\right)$ y en el punto de marchitamiento. Cuando el IDH es negativo, el suelo está sometido a déficit hídrico, que es absoluto cuando se alcanza el valor de $-100 \%$, situación con la que se llega al punto de marchitamiento. Valores positivos de IDH indican condiciones de exceso de agua.

En la figura 3 se muestra la evolución del IDH entre abril de 2000 y diciembre de 2004. Se observan de manera clara los intervalos deficitarios y excedentarios. Durante los casi cinco años de mediciones se ha constatado que, coincidiendo con el inicio del ciclo vegetativo del melojo, el suelo entra en situación de déficit hídrico (IDH $<0 \%$ ). Ese dato no sería muy llamativo si los valores se mantuvieran próximos a cero. Eso indicaría que el suelo dispondría de agua en condiciones de ser utilizada, a pesar de haberse reducido su contenido. Sin embargo, en todos los años se ha producido una caída muy pronunciada del IDH. Pero la circunstancia más destacada de este análisis es el hecho de que en verano el agua disponible para las plantas se agota completamente (IDH $\leq-100 \%$ ), y que esa situación se prolonga durante uno o dos meses consecutivos (agosto-septiembre) cada año. La reducción en la aportación debida a una menor precipitación y a la interceptación, unido al incremento de la evaporación y a la extracción de agua por el bosque, dejan al suelo exhausto.

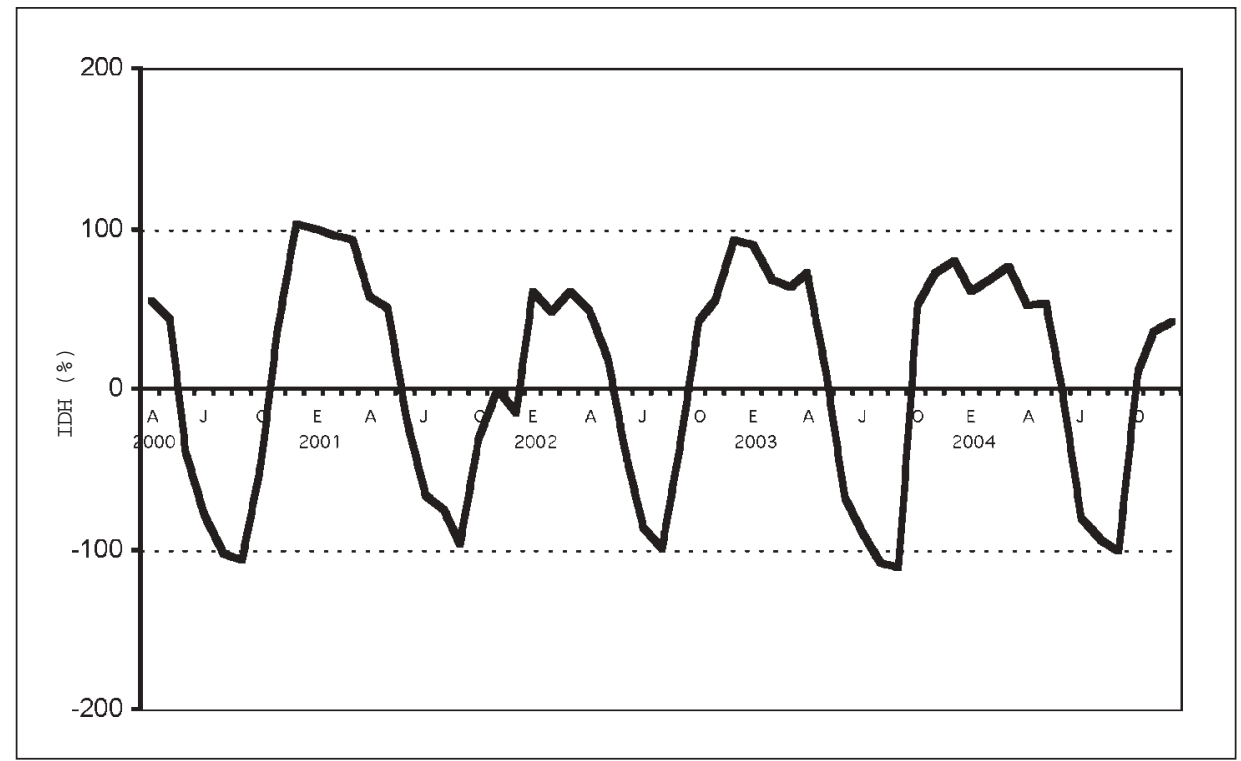

Figura 3. Evolución mensual del Índice de Déficit Hídrico (IDH) medio de los suelos de la cuenca experimental de Rinconada (Abril 2000 - Diciembre 2004). 


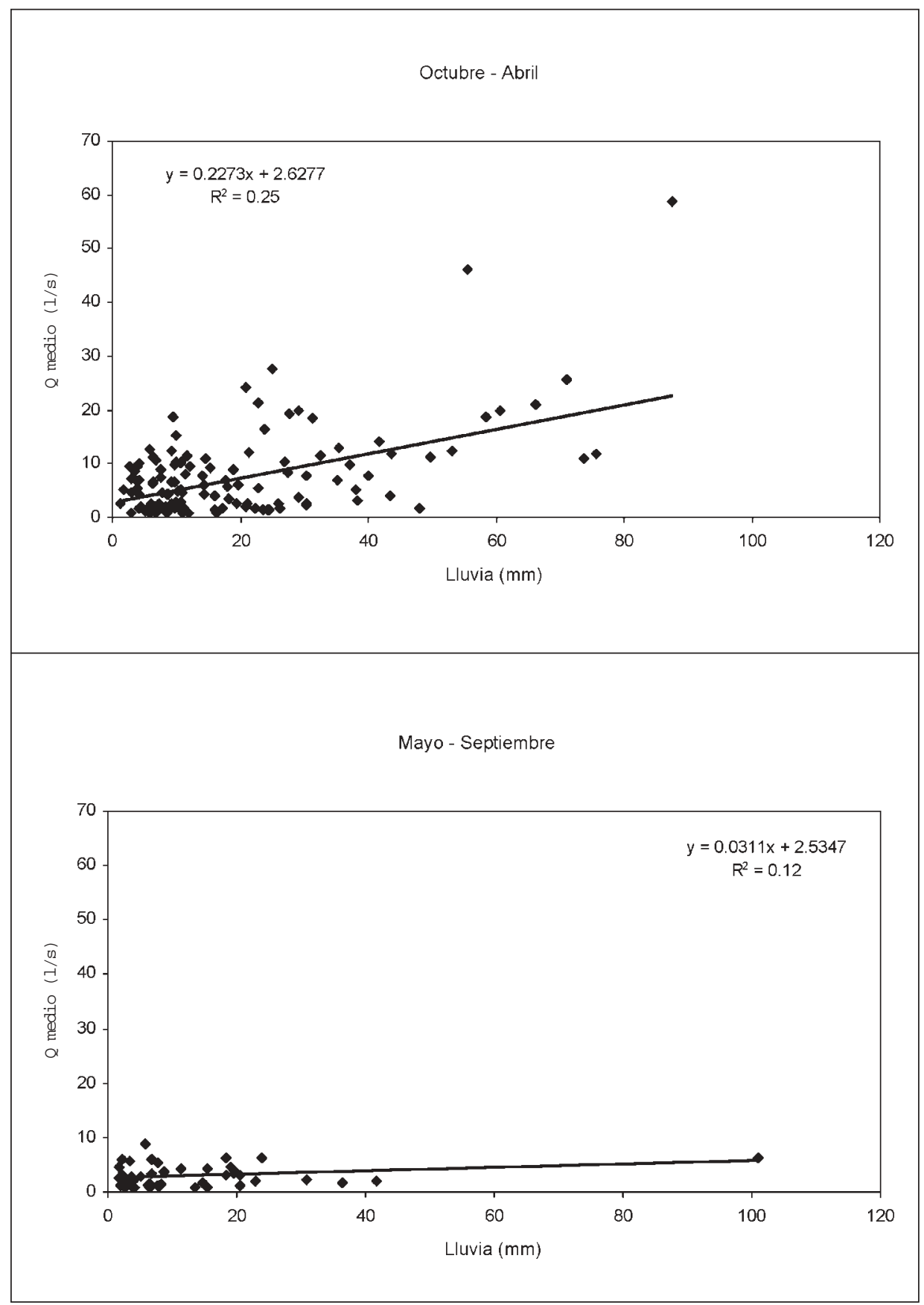

Figura 4. Relación entre el caudal medio diario y la precipitación diaria, correspondiente a los episodios de escorrentía del periodo enero 2000 a octubre 2003, durante la estación húmeda (octubre-abril) y seca (mayo-septiembre). 
El agotamiento del agua del suelo por el bosque se ha puesto de manifiesto mediante el seguimiento del contenido de agua de los árboles. En un trabajo reciente (Hernández Santana, 2005) se ha demostrado que el robledal objeto de estudio se ve sometido a un elevado nivel de estrés hídrico. Los árboles llegan al extremo de consumir agua de su depósito interno cuando el suelo alcanza esos niveles de déficit hídrico.

Esta situación tiene implicaciones en el comportamiento de la escorrentía de la cuenca. Se ha observado que a lo largo del periodo de déficit de agua en el suelo, la cuenca tiene una baja capacidad de generar escorrentía durante los episodios de lluvia. Cuando el agua en el sistema suelo-bosque se agota, la precipitación de cada evento (a veces intensa) es absorbida por la recarga incompleta del suelo y la capacidad para producir escorrentía se reduce al mínimo o es inexistente.

La precipitación que se registra en el periodo deficitario (mayo-septiembre) está en torno a $200 \mathrm{~mm}$ (222 mm en el pueblo de Rinconada, $186 \mathrm{~mm}$ en la cuenca), es decir, el $20 \%$ de la precipitación anual. Sin embargo, en ese mismo intervalo de tiempo se genera únicamente el $6 \%$ de la escorrentía producida por los episodios lluviosos (escorrentía rápida). En la figura 4 se muestra la relación entre precipitación diaria y caudal medio diario para los episodios registrados en la C.E. de Rinconada entre enero de 2000 y octubre de 2003, después de separar los eventos de la estación deficitaria y los del resto del año. La diferencia entre ambos periodos es muy marcada, no ya en valores absolutos que resultaría obvio, sino en términos relativos, es decir, en la capacidad para generar escorrentía. Las diferencias en la pendiente de las rectas de ajuste de ambas series ponen en evidencia el diferente comportamiento hidrológico. La baja significación en la correlación entre ambas variables durante el periodo deficitario abunda aún más en esa circunstancia.

\subsection{Mecanismos de generación de la escorrentía}

La C.E. de Rinconada presenta escorrentía permanente y su componente principal es el flujo subterráneo. Una vez separado el flujo base de la escorrentía producida por los episodios lluviosos, se ha estimado que el primero es el responsable del $90 \%$ del agua que exporta la cuenca (Tabla 2). En la figura 5 se muestra la evolución del caudal medio mensual desde enero de 2000 a diciembre de 2004. Se observa el escaso peso que tiene la escorrentía rápida frente al flujo base. Únicamente en los periodos muy húmedos (noviembre 2000 - marzo 2001, 1104 mm; septiembre 2002 - febrero 2003, $1053 \mathrm{~mm}$ ), dicha componente de la escorrentía total tiene un protagonismo mayor. En el primero de los dos periodos referidos supuso el $21 \%$ y en el segundo el $16 \%$ del agua que salió de la cuenca.

En términos generales, la cuenca genera poca escorrentía, máxime si se consideran factores como las condiciones pluviométricas y topográficas de la zona. El coeficiente global de escorrentía es del $17 \%$. Se trata de un valor bajo, sobre todo si se tiene en cuenta que en su mayor parte es debido al flujo subterráneo. Si se analiza únicamente la escorrentía generada directamente por los episodios lluviosos (Tabla 2), el coeficiente de 
Tabla 2. Características de la escorrentía en la cuenca experimental de Rinconada (2000-2004)

\begin{tabular}{|c|c|c|c|c|c|}
\hline $\begin{array}{c}\text { Escorrentía } \\
\text { total }\end{array}$ & $\begin{array}{c}\text { Precipitación } \\
(\mathrm{mm})\end{array}$ & $\begin{array}{l}\text { Q total } \\
(1 / s)\end{array}$ & $\begin{array}{c}\text { Q específico } \\
\left(1 / \mathrm{s} / \mathrm{km}^{2}\right)\end{array}$ & $\begin{array}{c}\text { Aportación } \\
(\mathrm{mm})\end{array}$ & $\begin{array}{l}\text { C E } \\
(\%)\end{array}$ \\
\hline $2000 *$ & 1038.0 & 1.6 & 2.6 & 78.9 & 7.6 \\
\hline 2001 & 976.6 & 4.7 & 7.5 & 236.8 & 24.2 \\
\hline 2002 & 1291.2 & 2.2 & 3.6 & 113.2 & 8.8 \\
\hline 2003 & 1215.6 & 5.5 & 8.9 & 279.1 & 23.0 \\
\hline 2004 & 649.9 & 2.4 & 3.9 & 124.5 & 19.2 \\
\hline Media & 1034.3 & 3.3 & 5.3 & 166.5 & 16.6 \\
\hline $\begin{array}{l}\text { Separación de } \\
\text { la escorrentía }\end{array}$ & $\begin{array}{l}\mathrm{Qb} \\
(1 / \mathrm{s})\end{array}$ & $\begin{array}{l}\text { Qe } \\
(1 / s)\end{array}$ & $\mathrm{Qb}(\%)$ & Qe $(\%)$ & $\begin{array}{c}\text { C E } \\
\text { Qe }(\%)\end{array}$ \\
\hline $2000^{*}$ & 1.4 & 0.2 & 85.3 & 14.7 & 1.0 \\
\hline 2001 & 4.3 & 0.4 & 91.4 & 8.6 & 2.1 \\
\hline 2002 & 1.9 & 0.3 & 85.3 & 14.7 & 1.3 \\
\hline 2003 & 5.1 & 0.4 & 92.5 & 7.5 & 1.8 \\
\hline 2004 & 2.4 & 0.1 & 97.7 & 2.3 & 0.4 \\
\hline Media & 3.0 & 0.3 & 90.4 & 9.6 & 1.3 \\
\hline
\end{tabular}

* Sin contabilizar los datos de caudal de mayo y junio, por avería del limnígrafo Q, caudal; C E, coeficiente de escorrentía; Qb, flujo base; Qe, escorrentía rápida.

escorrentía baja hasta el $1.3 \%$.

A pesar de que se registren episodios de lluvia de consideración, la respuesta de la cuenca es, en la mayoría de los casos, bastante modesta. Se ha analizado la relación entre la precipitación de los eventos $(\mathrm{N}=260)$ y el caudal que generan (Fig. 6), y se ha observado que existe una muy buena relación entre ambas variables (coeficiente de determinación $\mathrm{R}^{2}=0.89$ ). Sin embargo, es bastante notoria la necesidad de que la cantidad de lluvia supere un determinado umbral (30 $\mathrm{mm}$ aprox.) para que la cuenca comience a reaccionar de manera apreciable. En cualquier caso, se observa que los valores de escorrentía son extremadamente bajos, en relación con la magnitud de la precipitación que los genera.

Para profundizar más en el conocimiento de un comportamiento tan peculiar, se llevó a cabo un análisis de correlación entre variables relativas a la escorrentía (escorrentía total, escorrentía rápida, caudal máximo, coeficiente de escorrentía) y variables relacionadas con los episodios pluviométricos (cantidad de lluvia, intensidad, duración) y el estado del sistema en el momento de producirse el episodio (caudal previo). El resultado de este análisis demostró que la cantidad de lluvia tiene en todos los casos un elevado coeficiente de correlación con las variables de escorrentía. La intensidad de la precipitación, a pesar de que muestra una correlación estadísticamente significativa, presenta siempre coeficientes 


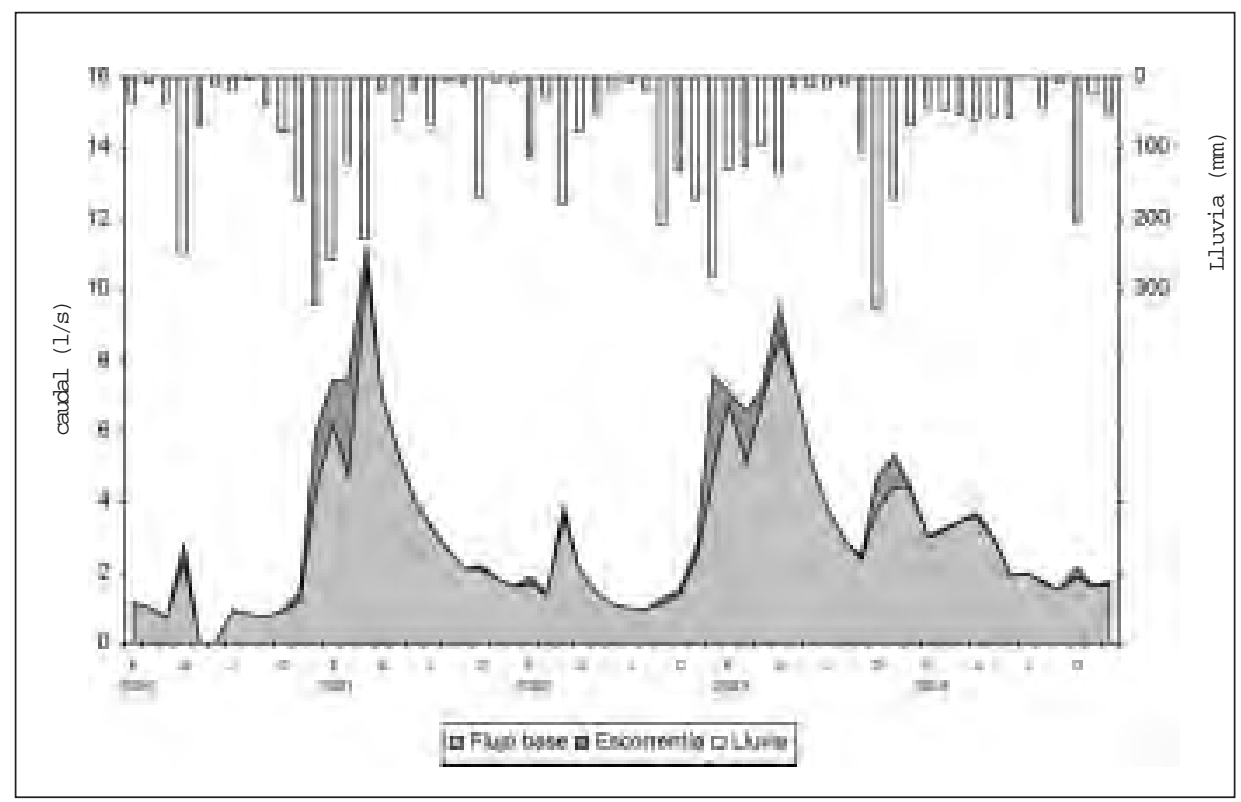

Figura 5. Hidrograma correspondiente a los valores medios mensuales de escorrentía y precipitación mensual, en el que se ha separado el flujo base de la escorrentía generada por los episodios de precipitación (escorrentía rápida), para el periodo entre enero de 2000 y diciembre de 2004. No aparecen los datos de mayo y junio de 2000 por avería en la estación de aforos.

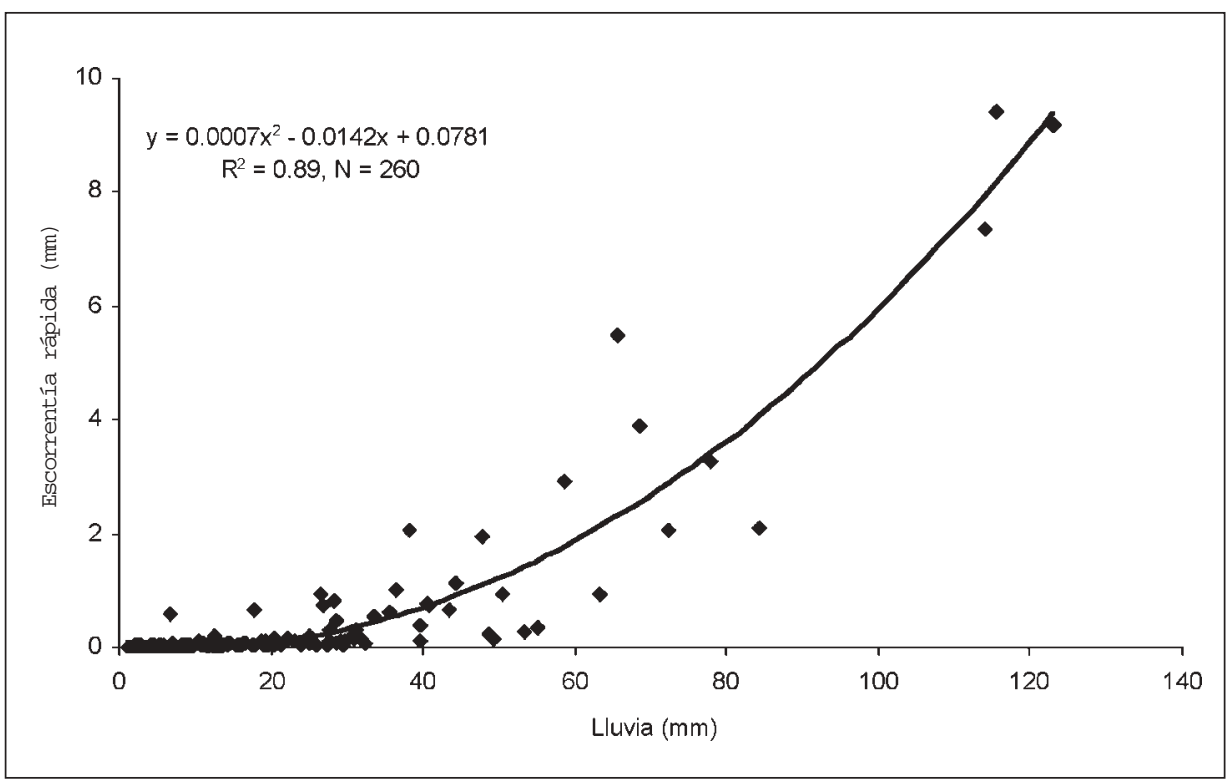

Figura 6. Relación entre precipitación y escorrentía generada por los episodios lluviosos (escorrentía rápida o escorrentía total menos flujo base) en la C.E. de Rinconada. 


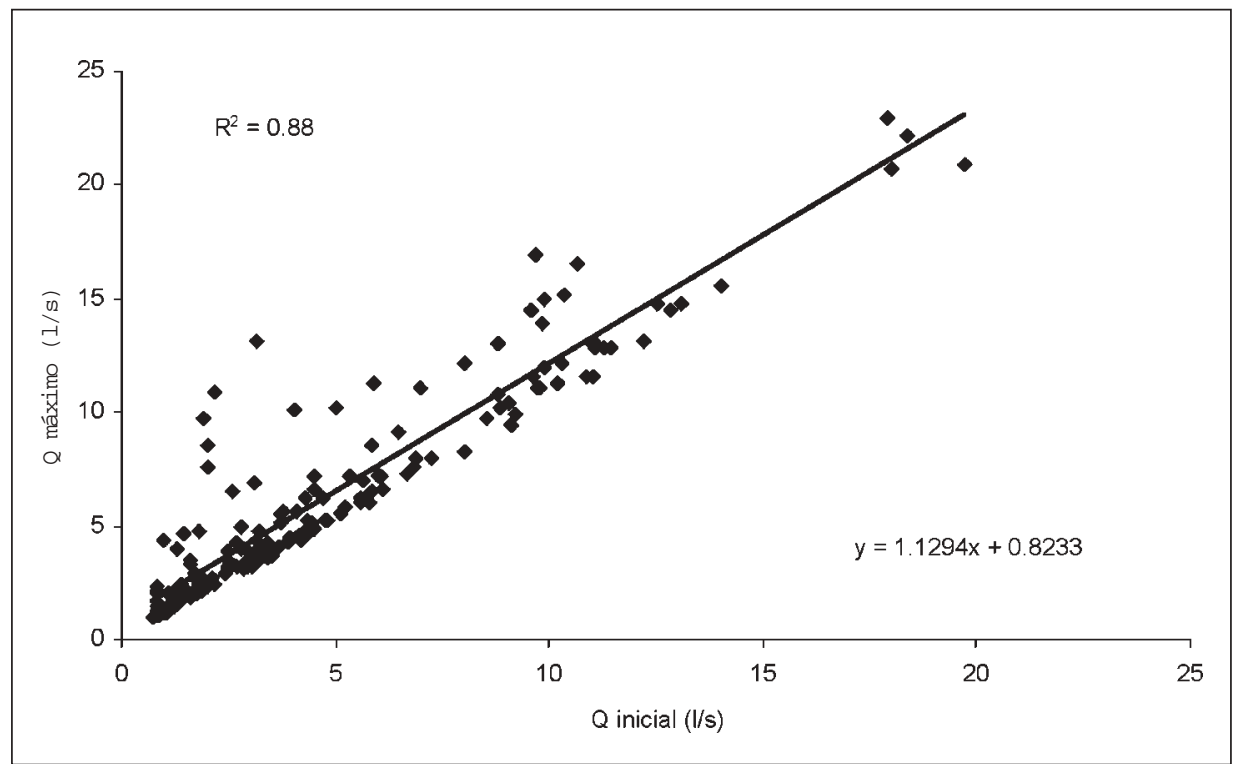

Figura 7. Relación entre caudales máximos de los eventos y caudales iniciales o previos al episodio de escorrentía (flujo base).

más bajos. Quizá el hecho más reseñable haya sido el elevado grado de correlación del caudal previo (flujo base) con las variables de escorrentía que definen los episodios.

A pesar de presentar un coeficiente de correlación muy significativo, el papel del caudal previo a la crecida de cada episodio queda diluido cuando se consideran al mismo tiempo todos los episodios. Parece razonable pensar que la respuesta de la cuenca será diferente ante eventos de larga (días) o corta (horas) duración. En el primer caso, la recarga del sistema es más efectiva, intervienen más factores y la respuesta se difiere en el tiempo. En el segundo, la reacción es más inmediata y, probablemente, sea más fácil acotar los mecanismos responsables.

Por esta razón se seleccionaron los episodios de duración inferior a 12 h $(\mathrm{N}=185$, $71 \%$ del total) y se analizó el papel del caudal previo en la generación de la escorrentía rápida. En la figura 7 se observa que existe una relación directa entre el caudal que circula en el momento de iniciarse el episodio y el caudal máximo que se alcanza. Del análisis de regresión se obtiene un coeficiente de determinación muy alto $\left(\mathrm{R}^{2}=0.88\right)$. Es importante también reseñar la escasa pendiente de la recta de regresión (1.13), lo que pone de manifiesto una vez más la débil respuesta de la cuenca en todos lo casos.

La estrecha vinculación entre escorrentía rápida y flujo base hay que relacionarla con el papel que desempeñan las zonas saturadas en el comportamiento hidrológico de la cuenca. No es frecuente encontrar bajo condiciones ambientales mediterráneas mecanismos de generación de escorrentía relacionados con flujo saturado o áreas fuente 
variables (Grésillon y Taha, 1998). Dichos mecanismos son propios de regiones de clima húmedo, ya sea en la zona templada (Carey y Woo, 2001; Buttle et al., 2004) o en las bajas latitudes (Putty y Prasad, 2000). En España se han estudiado esos procesos hidrológicos en cuencas de montaña pirenaicas (Gallart et al., 1997; Latron et al., 2004), pero bajo condiciones climáticas no estrictamente mediterráneas.

En la C.E. de Rinconada se ha hecho un estudio detallado de las zonas saturadas del fondo del valle, mediante control cartográfico, y se ha puesto de manifiesto el destacado protagonismo que tienen en la generación de escorrentía. En esta cuenca, debido a su configuración topográfica, las superficies que se saturan se encuentran en las proximidades del cauce y, casi siempre, están conectadas con él. El análisis de regresión entre el flujo base y la superficie saturada (Fig. 8) muestra que existe una relación directa y altamente significativa $\left(\mathrm{R}^{2}=0.97, \mathrm{~N}=14\right)$. Resulta muy sorprendente comprobar que las zonas saturadas contributivas suponen un porcentaje muy pequeño de la superficie de la cuenca. El máximo de superficie se alcanzó en el invierno 2000-2001. Durante los meses previos (noviembre a enero) se registraron $752 \mathrm{~mm}$ de lluvia, la cifra más alta para ese periodo en la serie de la cuenca experimental y superada en sólo dos ocasiones en la estación del pueblo de Rinconada de la Sierra en los últimos 50 años. A pesar de esas condiciones extraordinariamente húmedas, la superficie saturada máxima supuso el $0.31 \%$ de la superficie total de la cuenca.

La escasa capacidad de generar escorrentía, incluso con eventos de precipitación

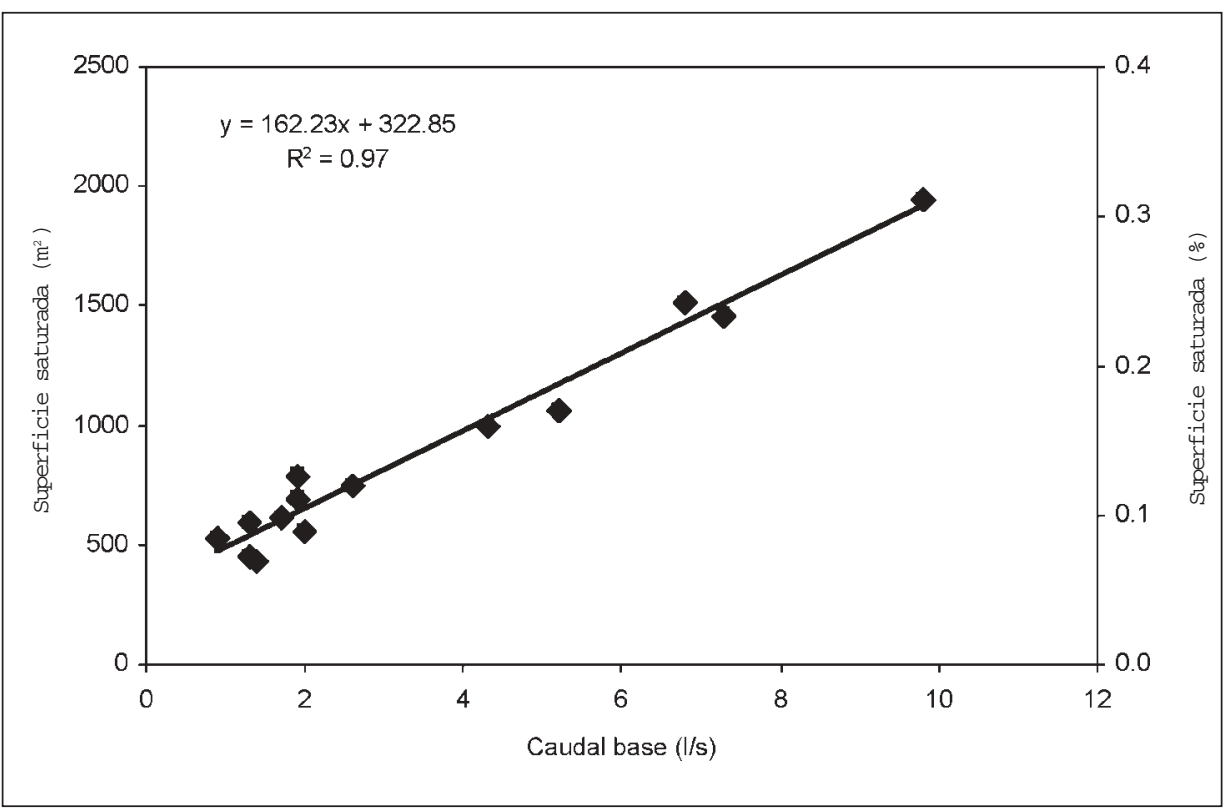

Figura 8. Relación entre flujo base y superficie saturada, expresada en términos absolutos y en porcentaje respecto al total de la cuenca experimental de Rinconada. 
considerables (Fig. 6), y la relación existente entre flujo base y caudal máximo (Fig. 7), pueden tener una explicación en el papel de las zonas saturadas contributivas. En la mayor parte de los episodios, las únicas superficies que aportarían escorrentía serían aquellas que se encuentran saturadas en las inmediaciones del cauce. El área que ocupan dichas superficies, aún siendo variable dependiendo de la estación y de la precipitación precedente, supone siempre un porcentaje sumamente pequeño del total de la cuenca. Eso supondría que, durante esos eventos, el volumen de agua que es exportada por la C.E. de Rinconada, estaría en relación exclusivamente con el volumen precipitado sobre las zonas saturadas. De hecho, el flujo por exceso de infiltración (hortoniano) únicamente se ha observado en sectores reducidos de la parte inferior de la cuenca, allí donde no hay bosque, los suelos son menos profundos y hay abundantes afloramientos rocosos.

\section{Conclusiones}

En este trabajo se ha puesto de manifiesto el papel tan destacado que tiene el bosque en la regulación de los flujos hídricos en una cuenca. A pesar de situarse en un sector bajo condiciones climáticas mediterráneas subhúmedas, con una precipitación anual abundante, la cuenca tiene una muy baja capacidad de producir escorrentía. La presencia de caudal permanente (flujo base) y una aparente abundante pluviometría, enmascaran una notable escasez de escorrentía superficial. La mediterraneidad de la zona, sobre todo la reducción de la precipitación en el verano, la elevada evapotranspiración, y el papel del bosque (interceptación y consumo de agua), condicionan sobremanera el comportamiento hidrológico de la cuenca.

En la C.E. de Rinconada se ha detectado la importancia que tienen el flujo saturado y las áreas fuente variables, en la respuesta de la escorrentía frente a los episodios lluviosos. Se trata de mecanismos propios de condiciones más húmedas de otras latitudes y, por ello, han sido poco estudiados bajo condiciones bioclimáticas mediterráneas. Esta constatación conduce a la necesidad de que se investigue más en estos aspectos, pues podrían tener un gran protagonismo en el comportamiento hidrológico de las cuencas de cabecera en muchos sectores montañosos del sur de Europa.

\section{Agradecimientos}

Los autores quieren expresar su agradecimiento al Ministerio de Ciencia y Tecnología (Proyectos REN2000-1157 y REN2003-00381), a la Junta de Castilla y León (Proyectos SA55-00A y SA016-03) y al Ministerio de Medio Ambiente (Red RESEL), sin cuya financiación no habría sido posible la realización de este trabajo. 


\section{Referencias bibliográficas}

ANDRÉASSIAN, V. (2004). Waters and forests: from historical controversy to scientific debate. Journal of Hydrology, 291: 1-27.

Beguería, S., LóPez-Moreno, J.I., Lorente, A., Seeger, M., y García-Ruiz, J.M. (2003). Assessing the effect of climate oscillations and land-use changes on streamflow in the Central Spanish Pyrenees. Ambio, 32: 283-286.

BENT, G. C. (2001). Effects of forest-management activities on runoff components and groud-water recharge to Quabbin Reservoir, central Massashusetts. Forest Ecology Management, 143: 115-129.

Beschta, R.L., Pyles, M.R., Skaugset, A.E., y Surfleet, C.G. (2000). Peakflow responses to forest practices in the western cascades of Oregon, USA. Journal of Hydrology, 233: 102-120.

BosCH, J.M. y HewLETT, J.D. (1982). A review of catchment experiments to determine the effect of vegetation on water yield and evapotranspiration. Journal of Hydrology, 55: 3-23.

Buttle, J.M.; Dillon, P.J. y Eerkes, G.R. (2004). Hydrologic coupling of slopes, riparian zones and streams: an example from the Canadian Shield. Journal of Hydrology, 287: 161-177.

CAREY, S.K. y WoO, M. (2001). Slope runoff processes and flow generation in a subartic, subalpine catchment. Journal of Hydrology, 253: 110-129.

CASSEl, D.K., KACHANOSKI, R.G. y ToPP, G.C. (1994). Practical consideration for using a TDR cable tester. Soil Technology, 7: 113-126.

Ceballos, A., Martínez-Fernández, J. y Luengo-Ugidos, M.A. (2004). Analysis of rainfall trends and dry periods on a pluviometric gradient representative of Mediterranean climate in the Duero basin, Spain. Journal of Arid Environments, 58: 214-232.

Cosandey, C., Andréassian, V., Martin, C., Didon-LEscot, J.F., Lavabre, J., Folton, N., MAthys, N. y Richard, D. (2005). The hydrological impact of the Mediterranean forest: a review of French research. Journal of Hydrology, 301: 235249.

De luis, M., Raventós, J., González-Hidalgo, J.C., SÁnchez, J.R. y Cortina J. (2000). Spatial analysis of rainfall trends in the region of Valencia (East Spain). International Journal of Climatology, 20: 1451-1469. 
JOFFRE, R. y RAMBAL, S. (1993). How tree cover influences the water balance of Mediterranean rangelands. Ecology, 74, 570-582.

Gallart, F., LATron, J., Llorens, P. y RABADÁ, D. (1997). Hydrological functioning of Mediterranean mountain basins in Vallcebre, Catalonia: some challenges for hydrological modelling. Hydrological Processes, 11: 1263-1272.

Gallart, F. y Llorens, P. (2004). Observations on land cover changes and water resources in the headwaters of the Ebro catchments, Iberian Peninsula. Physics and Chemistry of the Earth, 29: 769-773.

García-Ruiz. J.M., Arnáez, J., Beguería, S., Seeger, M., Martí-Bono, C., Regües, D., LANA-RENAULT, N. y WHite, S. (2005). Runoff generation in an intensively disturbed, abandoned farmand catchment, Central Spanish Pyrenees. Catena, 59: 79-92.

GRÉSILlON, J.M. y TAHA, A. (1998). Les zones saturées contributives, en climat méditerranéen: conditions d'aparition et influence sur les crues. Hydrological Science Journal, 43: 267-282.

HERNÁNDEZ SANTANA, V. (2005). Estimación del contenido de agua en árboles mediante TDR para el estudio del estrés hídrico: aplicación a Quercus pyrenaica Willd. Tesina de Licenciatura. Universidad de Salamanca. 86 pp.

Hernández Santana, V., Martinez Fernandez, J. y Ceballos Barbancho, A. (2004). Medición del contenido de humedad en árboles mediante TDR para el control del estrés hídrico: aplicación a Quercus pyrenaica Willd. Actas del III Congreso Español de Biogeografía. Urdaibai. (en prensa)

Instituto Nacional de Meteorología (1994). Calendario Meteorológico 1993. Instituto Nacional de Meteorología, Madrid.

IROUMÉ, A., Huber, A. y SCHUlz, K. (2005). Summer flows in experimental catchments with different forest covers, Chile. Journal of Hydrology, 300: 300-313.

LATron, J., Llorens, P. y GALlart, F. (2004). Dinámica estacional de las áreas fuente de escorrentía en una cuenca mediterránea de montaña (Vallcebre, Pirineos catalanes). En: Riesgos naturales y antrópicos en Geomorfología (G. benito y DíezHerrero, A., Eds.). Sociedad Española de Geomorfología - CSIC, Vol. II, pp. 149158, Madrid.

LóPez Gómez, A. (1989). El clima. En: Geografía General de España (de Terán, M., Solé Sabarís, L y Vilá Valentí, J. Coord.). Editorial Ariel, pp. 139-170, Barcelona.

MARTíN-VIDE, J. y GóMEZ, L. (1999). Regionalization of peninsular Spain based on the length of dry spells. International Journal of Climatology, 19: 537-555. 
Martínez FernándeZ, J. y Ceballos, A. (2001). Diseño y validación de una sonda TDR para la medición de la humedad del suelo. En: Temas de investigación en zona no saturada (López, J.J. y Quemada, M., eds.). Universidad Pública de Navarra, pp. 37-43, Pamplona.

Ministerio De Medio Ambiente (2000). Estrategia forestal española. Dirección General de Conservación de la Naturaleza, 240 pág., Madrid..

PUTTY, M.RY. y PRASAD, R. (2000). Understanding runoff processes using a watershed model: a case study in the Western Ghats in South India. Journal of Hydrology: 228, 215-227

RAMBAL, S. (1987). Évolution de l'occupation des terres et ressources en eau en région Méditerranéenne karstique. Journal of Hydrology, 93: 339-357.

Schnabel, S. y MATEOS, B. (2000). Hidrología superficial en ambientes adehesados, cuenca experimental de Guadalperalón. Cuadernos de Investigación Geográfica, 26: 113-130.

Sun, G., Mcnulty, S.G., Lu, J., Amatya, D.M., Liang, Y. y Kolka, R.K. (2004). Regional annual water yield from forest lands and its response to potential deforestation across the southeastern United States. Journal of Hydrology: doi:10.1016/j.jhydrol.2004.11.021.

TOPP, G.C., DAVIS, J.L. y ANNAN, A.P. (1980). Electromagnetic determination of soil water content: measurement in coaxial transmission lines. Water Resources Research, 16: 574-582.

Zegelin, S.J., White, I. y Russel, G.F. (1992). A critique of the Time Domain Reflectometry technique for determining field soil-water content. En: Advances in measurement of soil physical properties: bringing theory into practice. Soil Science Society American Special Pub. No. 30. 187-208. 